\title{
Araneofauna (Arachnida: Araneae) de suelo en bosques nativos, exóticos y pajonales del Parque General San Martín, Entre Ríos Argentina
}

\author{
Melina S. AlmadA ${ }^{1,2} \bowtie$ Juan A. Sarquis ${ }^{3}$ \\ ${ }^{1}$ Estación Experimental Agropecuaria INTA Reconquista, Santa Fe, Argentina. ${ }^{2}$ CONICET. ${ }^{3}$ Instituto Nacional de \\ Limnología (CONICET-UNL). Ciudad Universitaria, Santa Fe, Argentina.
}

\begin{abstract}
Resumen. La invasión de especies leñosas exóticas en la región argentina del Espinal provoca un cambio en la fisonomía del paisaje ya que altera el ambiente y produce la pérdida de biodiversidad. Se estudió la composición de arañas asociada al suelo del Parque General San Martín, Entre Ríos, durante el verano e invierno de 2012. Se seleccionaron tres ambientes: pajonal $(\mathrm{P})$, bosque nativo $(\mathrm{BN})$ y bosque exótico (BE), y se utilizaron trampas de caídas para el muestreo. Se recolectaron en total 1398 arañas (26 familias y 120 especies/morfoespecies). Las familias más abundantes en los tres ambientes fueron Lycosidae $(n=574 ; 41.06 \%)$, Tetragnathiidae $(n=223$; $15.95 \%)$ y Linyphiidae $(n=137 ; 9.80 \%)$, respectivamente. Los gremios que presentaron la mayor abundancia fueron "cazadoras corredoras sobre suelo" ( $n=632)$, "tejedoras de telas en sábanas" (n=262) y "tejedoras de telas orbiculares" ( $\mathrm{n}=244)$, representados en todos los ambientes estudiados. Los perfiles de diversidad indicaron que el BN fue más diverso que el $\mathrm{P}$ y el BE. Los valores de complementariedad mostraron una diferencia de $71 \%$ entre P-BE y P-BN, y de $63 \%$ entre BE-BN. Este trabajo constituye el primer registro sobre la comunidad de arañas de suelo del Parque General San Martín.
\end{abstract}

[Palabras clave: diversidad, arañas epígeas, parque provincial, invasiones]

\begin{abstract}
Ground aracnofauna (Araneae: Araneae) in native and exotic Forest and grasslands of San Martín Park, Entre Ríos, Argentina. The Exotic plant species invasion in the Argentine Espinal region causes a change in the appearance of the landscape, altering the environment and producing a significant loss of biodiversity. We studied the composition of ground spiders in Parque General San Martín, Entre Ríos, during the summer and winter seasons of 2012. Three areas were selected: pajonal (P), native forest (BN) and exotic forest (BE), pitfalls were used for sampling. A total of 1398 spiders (26 families and 120 species/morphospecies) were collected. The most abundant families in the different areas were Lycosidae ( $n=574 ; 41.06 \%)$, Tetragnathiidae $(\mathrm{n}=223 ; 15.95 \%)$ and Linyphiidae $(\mathrm{n}=137 ; 9.80 \%)$. The guild category "ground hunters" $(\mathrm{n}=632)$ "sheet web weavers" $(n=262)$ and "orb webs weavers" $(n=244)$ were highly represented in all sampling areas. Diversity indices showed that $\mathrm{BN}$ was more diverse than $\mathrm{P}$ and $\mathrm{BE}$. Complementarity values obtained between $\mathrm{P}$ and $\mathrm{BE}$ and $\mathrm{BN}$ were higher than $71 \%$, while $\mathrm{BE}$ and $\mathrm{BN}$ showed values of $63 \%$. This study constitutes the first record of the spider's community of Parque General San Martín.
\end{abstract}

[Keywords: diversity, epigeous spiders, soil, provincial park, invasions]

\section{INTRODUCCIÓN}

Las arañas (Clase Arachnida, Orden Araneae) representan uno de los grupos faunístico más diversos del reino animal, con aproximadamente 43000 especies descriptas (World Spider Catalog 2016). Este grupo cosmopolita (se las encuentra en todos los continentes excepto en la Antártida) se destaca por su rol como depredador, lo que constituye una característica clave por su impacto en cualquier ecosistema terrestre (Grismado et al. 2014). Han ganado una aceptación amplia en los estudios ecológicos como indicadores de unidades ambientales ya que sus comunidades están influenciadas por el tipo de hábitat y por el patrón de uso de la tierra (Maelfait et al. 1990; Weeks and Holtzer 2000). Además, las arañas

Editora asociada: Adriana Salvo

$\triangle$ almadamelina@gmail.com forman parte de la comunidad de artrópodos del suelo y son un componente clave de los ecosistemas naturales y los agroecosistemas. Participan en la regulación de procesos como la fragmentación y la descomposición de la materia orgánica, el reciclado de nutrientes, además de modificar la estructura del suelo y regular la actividad de otros organismos más pequeños (Cardoso et al. 2011; Díaz Porres et al. 2014).

Los problemas ambientales de la Argentina son diversos y están provocando una irremediable pérdida de la biodiversidad (Brown et al. 2005). La ecorregión del Espinal constituye uno de los ambientes naturales argentinos con mayor grado de modificación y con menos representación en las áreas 
naturales protegidas, donde únicamente $0.2 \%$ está preservado y legalmente declarado (Bertonatti and Corcuera 2000; Berduc 2004). En la provincia de Entre Ríos, el avance de la frontera agrícola y la urbanización llevaron a reemplazar extensas áreas de bosques nativos por otros ecosistemas, lo cual altera la riqueza y la abundancia de especies (Orjuela and Jiménez 2004).

El Parque General San Martín (PGSM) es un parque provincial (Entre Ríos) que en los últimos 50 años ha sufrido un proceso paulatino de invasión de leñosas exóticas que modifican el hábitat natural y la fisionomía de los montes nativos presentes. Esta invasión genera una disminución de la estructura del hábitat y una menor complejidad estructural de la vegetación, lo que se traduce en menor cantidad de nichos posibles para las especies (Uetz 1991; Wise 1993; Ávalos et al. 2009; Berduc et al. 2010). Esto se corresponde con la hipótesis de heterogeneidad del hábitat, que establece que mientras más complejo es el ambiente, más cantidad de nichos pueden coexistir. Por ello, es posible una mayor cantidad de especies (Bazzaz 1975). En este contexto, y teniendo en cuenta que la diversidad de arañas está influenciada por los cambios que se generan en el ambiente, se espera que la comunidad de arañas sea mayor en ambientes nativos y baja en ambientes exóticos donde la heterogeneidad del hábitat es menor por la acción de las especies leñosas que invaden y modifican el ambiente.

El objetivo de este trabajo fue conocer la comunidad de arañas asociada al estrato de suelo en diferentes ambientes naturales de la provincia de Entre Ríos y evaluar si difieren de acuerdo a las características ambientales presentes en el Parque San Martín. En Argentina no se realizaron estudios referidos a la comunidad de arañas en ambientes que presentan composiciones vegetales y heterogeneidades distintas dentro de un parque provincial, por lo que el presente estudio es el primer antecedente científico sobre la composición y la diversidad de arañas asociadas al estrato de suelo, y sobre la estructura de sus gremios en ambientes nativos y exóticos del Parque General San Martín.

\section{Materiales y MÉTOdOS}

\section{Área de estudio}

El muestreo se realizó en el PGSM, cuyas 400 ha están comprendidas dentro del área natural protegida Parque Escolar Rural Enrique Berduc ( $31^{\circ} 40^{\prime} \mathrm{S}$ - 60²0' O), ubicada en el Departamento Paraná, provincia de Entre Ríos, Argentina. En cuanto a su fitogeografía, el parque se encuentra dentro de la provincia del Espinal. Su temperatura media oscila entre 7 y $10^{\circ} \mathrm{C}$ en invierno y entre 19 y $23{ }^{\circ} \mathrm{C}$ en verano. Las precipitaciones superan los 1100$1200 \mathrm{~mm}$ anuales, con valores mayores para los meses de verano (Pereyra 2003). En el PGSM se observan tres tipos de ambientes florísticos naturales: a) monte nativo con características semixerofíticas, caracterizado por la presencia de dos especies de algarrobo (Prosopis alba y Prosopis nigra), ñandubay (Prosopis affinis), tala (Celtis tala) y molle (Schinus longifolia.), entre otras especies, b) vegetación costera, representada porárboles como ceibo (Erythrina crista-galli),sauce(Salixhumboldtiana)ycurupí (Sapium haematospermum), y c) pajonales graminiformes y vegetación acuática anfibia y flotante asociada.

En la actualidad, el parque se encuentra invadido por especies leñosas exóticas. El monte nativo está siendo reemplazado por especies leñosas exóticas como acacia negra (Gleditsia triacanthos), mora (Morus nigra) y ligustro (Ligustrum lucidum), entre otras especies. Esto ha generado bosques altos caducifolios y cambios severos en la fisonomía del parque en los últimos 50 años (Berduc 2004; Berduc et al. 2010).

\section{Trabajo de campo}

La recolección de arañas de suelo se realizó durante las estaciones de verano (diciembre 2011, enero y febrero 2012) e invierno (junio, julio y agosto 2012), en tres momentos consecutivos por estación, separados 25-30 días entre sí. Se seleccionaron tres ambientes representativos del parque: pajonal $(\mathrm{P})$, bosque nativo (BN) y bosque exótico (BE), separados entre sí por no menos de $500 \mathrm{~m}$, y se escogieron cuatro sitios $(1,2,3,4)$ en cada ambiente, según la vegetación circundante (nativa para bosques nativos, exótica leñosas para bosques exóticos y graminiformes, vegetación acuática anfibia y flotante para pajonales). En cada sitio se colocaron cinco trampas de caídas (pitfall) al ras del suelo, distribuidas al azar y separadas entre sí por una distancia mínima de $10 \mathrm{~m}$. De esa manera se totalizó 12 sitios distribuidos por el PGSM, colocándose 180 trampas por estación.

Para el muestreo de las arañas de suelo se utilizaron trampas de caídas (pitfall), que 
consistieron en un recipiente de plástico con una capacidad de $500 \mathrm{cc}$ (11 cm de profundidad y $7 \mathrm{~cm}$ de diámetro superior), utilizando como conservante una solución salina (cloruro de sodio (kg):agua (L) en proporción 1:8, con gotas de detergente para romper la tensión superficial) y se mantuvieron activas por siete días en cada momento por estación.

\section{Actividades de laboratorio}

En el laboratorio, se procedió a la separación de arañas del resto de los artrópodos recolectados. Las arañas fueron identificadas hasta género y especie en los casos posibles, y clasificadas en gremios (Dippenaar-Schoeman and Jocqué 1997; Ramírez 1999; Cardoso et al. 2011; World Spider Catálogo 2016). Las arañas que no pudieron ser identificadas taxonómicamente fueron separadas en morfoespecies (msp.) y designadas con el nombre de la familia y un número $\left(\mathrm{n}^{\circ}\right)$ (Oliver and Beattie 1993). Las especies/morfoespecies fueron condicionadas y conservadas en alcohol al $70 \%$. Se utilizaron individuos adultos y subadultos para el análisis; sólo se incluyeron juveniles cuando se conocían los estadios de la especie. El material recolectado se encuentra depositado en la colección aracnológica del Museo Argentino de Ciencias Naturales "Bernardino Rivadavia" (MACN-Ar, Cristina L. Scioscia).

\section{Análisis de datos}

Para comparar la diversidad de arañas entre los ambientes se generaron perfiles de diversidad de Renyi (Tóthmérész 1995) mediante el programa PAST v2.16 (Hammer et al. 2012). Se estimó la diversidad verdadera (Jost 2006) mediante los números efectivos de especies. Se consideró la riqueza total de especies (diversidad verdadera de orden cero) utilizando el valor del estimador ACE (del inglés abundance-based coverage estimator), el exponencial del índice de Shannon (diversidad verdadera de orden 1), y el inverso del índice de Simpson (diversidad verdadera de orden 2) utilizando el estimador MVUE (del inglés minimum variance unbiased estimator). Los estimadores fueron calculados con el software SPADE (Chao and Shen 2009).

Se observó el grado de disimilitud de los ensambles de especies utilizando el porcentaje de complementariedad que mide el grado de diferencia en la composición de especies entre comunidades distintas (Colwell and Coddington 1994). El número de especies compartidas entre los ambientes se calculó con el software SPADE. Para probar si existieron diferencias estadísticas significativas entre las comunidades de arañas de los ambientes se realizó un análisis de similitud (ANOSIM) utilizando la matriz de abundancia de una vía, con una permutación de 9999 y con un nivel de significancia $P<0.05$. Además, para determinar qué especies de arañas contribuyen más a la disimilitud entre los ambientes se realizó un análisis de porcentaje de similitud (SIMPER) mediante el índice de similitud de Bray-Curtis, utilizando el programa PAST.

\section{Resultados}

Se recolectaron un total de 1398 arañas, agrupadas en 26 familias (38.3\% de las familias conocidas en Argentina). Del total de individuos, 1396 correspondieron a 25 familias del suborden Araneomorphae y sólo dos individuos pertenecieron a la Familia Nemesiidae, del suborden Mygalomorphae (Tabla 1). Respecto al estado de desarrollo de las arañas capturadas en todo el muestreo, $45 \%$ correspondió a juveniles, $34 \%$ a machos y $21 \%$ a hembras.

Se identificaron 120 especies / morfoespecies, destacándose Lycosa erythrognatha (Lucas 1836) (Lycosidae) como la más abundante (293 individuos; 20.96\%), seguida de la msp. Tetragnathidae $n^{\circ} 67$ (221 individuos; 15.81\%). La familia más abundante fue Lycosidae (574 individuos; $41.06 \%$ del total de arañas recolectadas), seguida por Tetragnathidae (223 individuos; 15.95\%) y Linyphiidae (137 individuos; $9.80 \%$ ). Clubionidae y Nephilidae (3y 1 individuo respectivamente) se registraron únicamente en el BE, Mimetidae y Sparassidae (1 y 2 individuos respectivamente) en el P y Nemesiidae ( 2 individuos) en BN. Las familias Amaurobiidae, Scytodidae y Thomisidae sólo se encontraron en los bosques; Oxyopidae y Philodromidae en $\mathrm{BE}$ y $\mathrm{P}$, mientras que Tetragnathidae se detectó en P y BN.

La diversidad de los ambientes comparada por medio de los perfiles de diversidad (Figura 1) mostró que el ambiente de bosque nativo (BN) fue más diverso (Tabla 2), 2.73 veces mayor que el ambiente $P$ y 2.18 que el BE al comparar los valores de D1 observado (diversidad de orden 1). Si bien la completitud de inventario fue muy buena a nivel general y para los pajonales, no lo fue para los ambientes de bosque nativo, donde faltó una proporción moderada de arañas por muestrear ya que los inventarios obtenidos fueron menores a $58 \%$. 
Tabla 1. Composición, abundancia y riqueza específica total de las familias de arañas de suelo registradas en el Parque General San Martín, agrupadas por gremios (Entre Ríos, Argentina). BE: bosque exótico; BN: bosque nativo; P: pajonales

Table 1. Composition, abundance and specific richness total of ground spider families in Park General San Martín, by guild (Entre Ríos, Argentina). BE: forest exotic; BN: forest native; P: grasslands.

\begin{tabular}{|c|c|c|c|c|c|c|c|}
\hline Gremios & Familia & $\begin{array}{l}\text { Riqueza } \\
\text { sp./msp. }\end{array}$ & $\mathrm{BN}$ & $\mathrm{P}$ & $\mathrm{BE}$ & Total & $\begin{array}{c}\text { Gremios } \\
\%\end{array}$ \\
\hline \multirow{3}{*}{ Cazadoras corredoras sobre el suelo } & Corinnidae & 8 & 8 & 13 & 3 & 24 & 45.21 \\
\hline & Gnaphosidae & 5 & 7 & 11 & 16 & 34 & \\
\hline & Lycosidae & 11 & 68 & 241 & 265 & 574 & \\
\hline Cazadoras deambuladoras & Thomisidae & 6 & 8 & 0 & 3 & 11 & 0.79 \\
\hline \multirow[t]{2}{*}{ Especialistas } & Mimetidae & 1 & 0 & 1 & 0 & 1 & 2.58 \\
\hline & Zodariidae & 1 & 11 & 2 & 22 & 35 & \\
\hline \multirow[t]{9}{*}{ Otras cazadoras } & Anyphaenidae & 10 & 16 & 6 & 5 & 27 & 12.59 \\
\hline & Clubionidae & 2 & 0 & 0 & 3 & 3 & \\
\hline & Ctenidae & 4 & 19 & 17 & 32 & 68 & \\
\hline & Miturgidae & 10 & 7 & 7 & 4 & 18 & \\
\hline & Oxyopidae & 1 & 0 & 3 & 4 & 7 & \\
\hline & Philodromidae & 1 & 0 & 2 & 1 & 3 & \\
\hline & Salticidae & 12 & 12 & 8 & 13 & 33 & \\
\hline & Scytodidae & 1 & 5 & 0 & 10 & 15 & \\
\hline & Sparassidae & 2 & 0 & 2 & 0 & 2 & \\
\hline \multirow[t]{4}{*}{ Tejedoras de telas en sábanas } & Amaurobiidae & 2 & 6 & 0 & 2 & 8 & 18.74 \\
\hline & Hahniidae & 2 & 1 & 95 & 12 & 108 & \\
\hline & Linyphiidae & 17 & 31 & 27 & 79 & 137 & \\
\hline & Pisauridae & 2 & 1 & 6 & 2 & 9 & \\
\hline \multirow[t]{3}{*}{ Tejedoras de telas espaciales } & Pholcidae & 1 & 1 & 2 & 5 & 8 & 2.5 \\
\hline & Theridiidae & 9 & 3 & 8 & 9 & 20 & \\
\hline & Titanoecidae & 2 & 2 & 2 & 3 & 7 & \\
\hline Tejedoras de telas irregulares & Nemesiidae & 1 & 2 & 0 & 0 & 2 & 0.14 \\
\hline \multirow[t]{3}{*}{ Tejedoras de telas orbiculares } & Araneidae & 6 & 9 & 4 & 7 & 20 & 17.45 \\
\hline & Nephilidae & 1 & 0 & 0 & 1 & 1 & \\
\hline & Tetragnathidae & 3 & 2 & 221 & 0 & 223 & \\
\hline \multicolumn{2}{|c|}{ Total } & 120 & 219 & 678 & 501 & 1398 & 100 \\
\hline
\end{tabular}

Los tres ambientes estudiados compartieron muy pocas especies (21), mostraron un valor alto de disimilitud $\left(1-\mathrm{C}_{33}=0.781\right)$ y alcanzaron valores de complementariedad de hasta $71 \%$ entre P-BE y P-BN, y de 63\% entre BE-BN. El $\mathrm{BN}$ fue el más disímil, con una similitud con BE de $40 \%$ y con P del $23.3 \%$. Los análisis de ANOSIM entre ambientes mostraron diferencias significativas en la composición de las comunidades entre P-BN y P-BE $(P<0.05$; $R=0.5949$ ), pero no entre los bosques. Las especies que mayor contribución realizaron a la disimilitud entre las comunidades de los ambientes, teniendo en cuenta los análisis de SIMPER, fue Tetragnathidae msp. 60, que proporcionó la mayor contribución entre los ambientes, seguida por Lycosa erythrognathay Alopecosarosea(Mello-Leitão1945),Lycosidae, Linyphiidae msp. 70 y Hahniidae msp. 63.

Se registraron ocho gremios en el PGSM. Las "cazadoras corredoras sobre suelo" $(n=632)$ fueron las más abundantes en todos los ambientes, seguido por las "tejedoras de telas en sábanas" ( $n=262)$ y las "tejedoras de telas orbiculares" $(n=244)$. En el BN se hallaron todos los gremios presentes en el parque, pero en el BE no se registró las "tejedoras de telas irregulares", al igual que las "cazadoras deambuladoras" en el P (Tabla 1). 


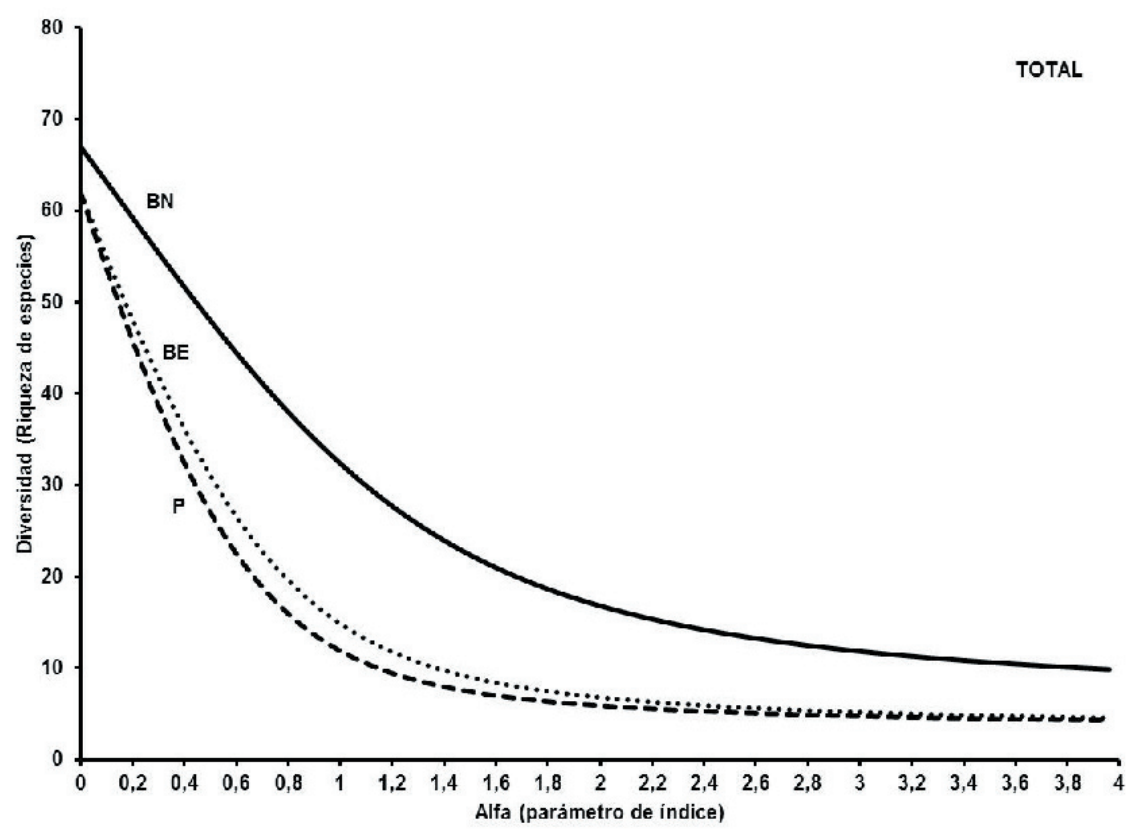

Figura 1. Perfiles de diversidad de arañas de suelo registradas en el Parque General San Martín (Entre Ríos, Argentina). BE: bosque exótico; $\mathrm{BN}$ : bosque nativo; $\mathrm{P}$ : pajonales.

Figure 1. Diversity profiles of ground spider in Park General San Martín (Entre Ríos, Argentina). BE: forest exotic; BN: forest native; P: grasslands.

Tabla 2. Diversidad observada y estimada de todo el muestreo y de cada ambiente, y el porcentaje de completitud de inventario teniendo en cuenta los valores de Sobs y ACE (abundance-based coverage estimator). BE: bosque exótico; $\mathrm{BN}$ : bosque nativo; P: pajonales.

Table 2. Observed and estimated diversity considering all the inventory and environment, and the percentage of the inventory completion taking in account the Sobs and the ACE value (abundance-based coverage estimator). BE: forest exotic; BN: forest native; P: grasslands.

\begin{tabular}{lccccccc}
\hline \multicolumn{1}{c}{ Ambientes } & \multicolumn{2}{c}{ Diversidad observada } & \multicolumn{3}{c}{ Diversidad estimada } & Completitud de \\
& ${ }^{0} \mathrm{D}$ & ${ }^{1} \mathrm{D}$ & ${ }^{2} \mathrm{D}$ & ${ }^{0} \mathrm{D}(\mathrm{ACE})$ & ${ }^{1} \mathrm{D}(\mathrm{MLE}-\mathrm{bc})$ & ${ }^{2} \mathrm{D}($ MVUE) & inventario (\%) \\
\hline Total & 120 & 22.76 & 10.55 & $165.4 \pm 14.3$ & $24.145 \pm 4.420$ & $10.62 \pm 0.20$ & 72.55 \\
$\mathrm{BN}$ & 67 & 32.36 & 16.79 & $145.8 \pm 29.6$ & $45.051 \pm 11.56$ & $18.11 \pm 0.24$ & 45.95 \\
$\mathrm{P}$ & 62 & 11.87 & 5.89 & $85.7 \pm 10.4$ & $12.639 \pm 2.606$ & $5.93 \pm 0.26$ & 72.35 \\
$\mathrm{BE}$ & 62 & 14.85 & 6.79 & $105.5 \pm 17.3$ & $16.483 \pm 4.289$ & $6.87 \pm 0.24$ & 58.77 \\
\hline
\end{tabular}

Diversidad observada y estimada: ${ }^{\circ} \mathrm{D},{ }^{1} \mathrm{D}$ y ${ }^{2} \mathrm{D}$ (indican medidas de diversidad de orden 0,1 , 2 , respectivamente). Diversidad observada: ${ }^{0} \mathrm{D}=$ Sobs (riqueza de especies observada); ${ }^{1} \mathrm{D}=$ exponencial del índice de Shannon; ${ }^{2} \mathrm{D}=$ inversa del índice de Simpson. Diversidad estimada: ${ }^{0} \mathrm{D}=\mathrm{ACE}$ (abundance-based coverage estimator); ${ }^{1} \mathrm{D}=$ bias-controled índice de Shannon; ${ }^{2} \mathrm{D}=\mathrm{MVUE}$ (mínimum variance unbiased estimator).

Observed and estimated diversity: ${ }^{0} \mathrm{D},{ }^{1} \mathrm{D}$ y ${ }^{2} \mathrm{D}$ (denote diversity measures of order 0,1 and 2 , respectively). Observed diversity: ${ }^{0} \mathrm{D}=$ Sobs (observed species richness); ${ }^{1} \mathrm{D}=$ exponential of Shannon index; ${ }^{2} \mathrm{D}=$ inverse of Simpson index. Estimated diversity: ${ }^{0} \mathrm{D}=\mathrm{ACE}$ (abundance-based coverage estimator); ${ }^{1} \mathrm{D}=$ biascontroled Shannon index; ${ }^{2} \mathrm{D}=\mathrm{MVUE}$ (minimum variance unbiased estimator).

\section{DISCUSIÓN}

Este trabajo representa el primer estudio sobre la composición de arañas de suelo del PGSM para la provincia de Entre Ríos y demuestra la alta diversidad presente en los diferentes ambientes del parque, donde las características y vegetaciones presentes en cada ambiente generan las condiciones adecuadas para el establecimiento de los diferentes ensambles de arañas. La araneofauna del suelo representó casi $40 \%$ de las familias (26 de 68) citadas para Argentina (World Spider Catálogo 2016) con 120 especies/msp., lo cual es significativo ya que sólo se corresponde a un estrato. En comparación con trabajos realizados en Sudamérica, encontramos resultados semejantes a lo encontrado por Costa et al. (2006) en Uruguay (88 especies; 25 familias) y menores a los registrados por Lopes y colaboradores (2006) en pastizales de Brasil $(20 ; 19)$. Si comparamos con otros trabajos de Argentina, en áreas naturales que abarcan otras escalas y períodos de muestreo y que incluyeron otras técnicas de toma de datos en más de un estrato, se observó un mayor número de especies y familias, como lo registrado en las Yungas del noroeste argentino (188 especies; 34 familias) (Rubio 2015), en el Parque Nacional Mburucuyá (Corrientes) (104; 28) (Rubio et al. 2008), en 
la Reserva Natural Otamendi (Buenos Aires) $(158 ; 32)$ (Grismado 2007) y en la Reserva Iberá (Corrientes) (152; 33) (Ávalos et al. 2009).

En cuanto a las familias de arañas registradas en el parque, Lycosidae (41\%) fue la más abundante, seguida por Tetragnathidae (16\%) y Linyphiidae (10\%). El resto de las familias no superan el 5\% de la abundancia total. Estas familias se corresponden con las especies que mayor contribución realizaron a la disimilitud entre los ambientes, donde las condiciones microclimáticas favorables que se generan (i.e., cobertura del suelo, refugio y disponibilidad de presas) establecen ambientes más propicios para el desarrollo de estas especies cazadoras y tejedoras de telas (Toft 1989).

Ávalos y colaboradores (2009) señalaron que la presencia conjunta de Linyphiidae y Lycosidae no supera el $45 \%$ en áreas protegidas, y alcanza hasta un 85\% en áreas degradadas. En nuestro estudio encontramos que en los bosques nativos y en los pajonales del PGSM, la presencia de Linyphiidae y Lycosidae no supera en conjuntos el $45 \%$, infiriendo el buen estado de estos ambientes. En cambio, en los bosques exóticos se halló la mayor proporción de licósidos y linífidos, superando el 65\%. Esto indicaría cierto grado de alteración por la invasión de especies leñosas en el parque, que modifica la fisionomía del paisaje e influye sobre la composición y la diversidad de las arañas (Churchill 1997).

Cardoso (2009) propone que el inventario debe ser razonable y que al menos $50 \%$ de las especies deben estar incluidas en los análisis. En el presente estudio, la completitud del inventario total y la de los ambientes pajonales fue buena (superior al 70\%) y menor en los bosques. Posiblemente, para mejorar y acrecentar el inventario de especies en los ambientes del parque se deberían incorporar otras técnicas de colecta, en otros períodos y estratos respecto a futuras investigaciones. En términos de conservación, es fundamental completar los inventarios parciales existentes para evaluar la biodiversidad y sus problemas de conservación a distintas escalas geográficas (Motta-Junior et al. 2008) ya que la riqueza de especies es una medida natural y simple para describir la comunidad y la diversidad regional (Magurran 1988; Jiménez-Valverde and Hortal 2003; Fandiño and Giraudo 2010).

Brown (2005) establece que la introducción de especies leñosas invasoras representa una amenaza para la diversidad biológica ya que altera los procesos ecosistémicos del suelo (i.e., ciclo de nutrientes y química del suelo, entre otros). En consecuencia, la disminución de las especies nativas cambia la fisionomía del ecosistema, lo que influye sobre la comunidad de arañas (que son sensibles a cambios del ambiente) y modifica su diversidad (Uetz 1991). Esto se observó en los perfiles de diversidad entre los ambientes, donde el BN fue más diverso que P y BE. Así mismo, los resultados del ANOSIM indicaron que los ambientes boscosos son diferentes a los pajonales, con una comunidad de arañas más diversa que responde a las características estructurales, fisonómicas y ecológicas del suelo (Díaz Porres et al. 2014).

Sólo 17.5\% de las especies fueron compartidas entre los ambientes, lo que indica altos valores de disimilitud y de alta complementariedad, preferentemente entre bosques nativos y pajonales. Las diferencias en la distribución de especies está correlacionada con la preferencia de diferentes tipos de sustratos (Wise 1993); por ello, las especies que aportaron a la disimilitud posiblemente sean arañas cuyas condiciones climáticas (humedad, temperatura) y requisitos ambientales sean diferentes entre los ambientes estudiados.

Se encontraron todos los gremios propuestos por Cardoso et al. (2011). El Parque muestra ser un área estructuralmente compleja, donde la vegetación baja representa un microhábitat para la coexistencia de los gremios "cazadoras sin tela" (Ctenidae, Lycosidae y Salticidae) y las típicas "tejedoras de telas" (Araneidae, Tetragnathidae) (Uetz 1991). La alta proporción de los gremios "cazadoras corredoras sobre el suelo", "tejedoras de telas en sábanas" y "tejedoras de telas orbiculares" en todos los ambientes se encuentran determinados por la extraordinaria abundancia de las familias Lycosidae, seguida de Linyphiidae y Tetragnathidae. Esto se relaciona directamente con la composición y la complejidad del paisaje (Roschewitz et al. 2005; Schmidt et al. 2008). Sin embargo, no se registró el gremio "tejedoras de telas irregulares" en el BE ni en $\mathrm{P}$, al igual que las "cazadoras deambuladoras" en este último ambiente. Esto refleja que la comunidad de arañas está muy influenciada por el tipo de hábitat y por los patrones de uso del suelo (Uetz 1991; Cardoso et al. 2011), siendo el BN el que ofrece mayor diversidad de nichos ecológicos para el establecimiento de los diferentes gremios (Weyland and Zaccagnini 2008).

Este trabajo conforma el primer estudio sobre la composición de arañas de suelo del Parque General San Martín, demostrando 
que la araneofauna del estrato bajo asociada a sitios con especies nativas es más diversa con un mayor número de gremios que ambientes con invasión de leñosas y pajonales. Nuestros resultados muestran la necesidad de aumentar los estudios tendientes a enriquecer los inventarios de especies y a conocer la biología y la ecología de un grupo depredador clave en los sistemas. Su evaluación ayudará a reforzar programas de conservación de áreas declaradas, como las del PGSM, que se encuentran débilmente conservadas.
Agradecimientos. Agradecemos a Alfredo Berduc por su colaboración y asesoramiento; a la Administración del Parque General San Martín, por permitirnos el acceso al mismo para realizar el estudio. A la FHUC-UNL (Facultad de Humanidades y CienciasUniversidad Nacional del Litoral) por su colaboración y CONICET (Consejo Nacional de Investigaciones Científicas y Técnicas) por su apoyo. A Luis Piacentini por su colaboración en la identificación taxonómica (MACN, Buenos Aires, Argentina).

\section{REFERENCIAS}

Ávalos, G., G. Rubio, M. Bar, M. Damborsky, and E. Oschorov. 2009. Composición y distribución de la araneofauna del Iberá. Rev Biol Trop 57(1-2):339-351.

Bazzaz, F. A. 1975. Plant species diversity in old-field successional ecosystems in southern Illinois. Ecology 56(2): 485-488.

Berduc, A. J. 2004. Diagnóstico y planificación de estrategia de manejo y control de leñosas exóticas en el Parque Escolar Rural "Enrique Berduc", CGE, La Picada, Entre Ríos. Consejo General de Educación de Entre Ríos. Pp. 28.

Berduc, A. J., P. L. Bierig, A. V. Donello, and C. H. Walker. 2010. Lista actualizada y análisis preliminar del uso de hábitat de medianos y grandes mamíferos en un área natural protegida del espinal con invasión de leñosas exóticas, Entre Ríos, Argentina. FABICIB vol 14:9-27.

Bertonatti, C., and J. Corcuera. 2000. Situación Ambiental Argentina 2000. Fundación Vida Silvestre Argentina. Buenos Aires. Pp. 436.

Brown, A., U. Martínez Ortíz, M. Acerbi, and J. Corcuera. 2005. La Situación Ambiental Argentina 2005, Fundación Vida Silvestre Argentina, Buenos Aires. Pp. 240-246.

Cardoso, P. 2009. Standardization and optimization of arthropod inventories-the case of Iberian spiders. Biodivers Conserv 18:3949-3962.

Cardoso, P., S. Pekár, R. Jocqué, and J. A. Coddington. 2011. Global Patterns of Guild Composition and Functional Diversity of Spiders. PLoS ONE 6(6):e21710. doi:10.1371/journal.pone.0021710.

Chao, A., and T. Shen. 2009. Program SPADE (Species Prediction and Diversity stimation). http://chao.stat.nthu.ed u.tw.

Churchill, T. 1997. Spiders as ecological indicators: an overview for Australia. Mem Mus Victoria 56(2):331-337.

Colwell, R., and J. Coddington. 1994. Estimating terrestrial biodiversity through extrapolation. Philosophical Transactions Royal Society B 345:101-118.

Costa, F., M. Simó, and A. Aisenberg. 2006. Composición y ecología de la fauna epígea de Marindia (Canelones, Uruguay) con especial énfasis en las arañas: un estudio de dos años con trampas de Intercepción. Pp. 427-436 en: R. Menafra, L. Rodríguez-Gallego, F. Scarabino and D. Conde (eds.). Bases para la conservación y el manejo de la costa uruguaya. Vida Silvestre Uruguay, Montevideo i-xiv. Pp. 668.

Díaz Porres, M., M. H. Rionda, A. E. Duhour, and F. R. Momo. 2014. Artrópodos del suelo: Relaciones entre la composición faunística y la intensificación agropecuaria. Ecol Austral 24:327-334.

Dippenaar-Schoeman, A. S., and R. Jocqué. 1997. African Spiders: An Identification Manual. Plant Protection Research Institute Handbook $\mathrm{N}^{\circ} 9$.

Fandiño, B., and A. R. Giraudo. 2010. Revisión del inventario de aves de la provincia de Santa Fe, Argentina. FABICIB vol 14:116-137.

Grismado, C. 2007. Comunidades de arañas de la Reserva Natural Otamendi, Provincia de Buenos Aires. Riqueza específica y diversidad. Trabajo de Seminario. Universidad CAECE, Buenos Aires, Argentina. Pp. 97.

Grismado, C. J., M. J. Ramírez, and M. A. Izquierdo. 2014. Araneae: Taxonomía, Diversidad y clave de identificación de familias de la Argentina. Pp: 55-94 en: S. Roig-Juñent, L. E. Claps and J. J. Morrone (eds.). Biodiversidad de Artrópodos Argentinos. Editorial INSUE - UNT, San Miguel de Tucumán, Argentina.

Hammer, O., D. A. T. Harper, and P. D. Ryan. 2012. PAST: Paleontological Statistics software package for education and data analysis. Paleontología Electrónica 4:1-9. Vers. 2.16. URL: http://palaeo-electronica.org/2001_1/past/ issue1_01.htm.

Jiménez-Valverde, A., and A. J. Hortal. 2003. Las curvas de acumulación de especies y la necesidad de evaluar la calidad de los inventarios biológicos. Rev Iber Arac 8:151-161.

Jost, L. 2006. Entropy and diversity. Oikos 113(2):363-375.

Lopes, J., F. Pereira dos Santos, and I. M. Medri. 2006. Araneofauna capturada no interior da mata e área de pastagem adjacente, no norte do Paraná, Brasil. Semina: Ciências Biológicas e da Saúde, Londrina, 27(2):133-138.

Maelfait, J. P., R. Jocqué, L. Baert, and K. Desender. 1990. Heathland management and spiders. Acta Zoologica Fennica 190:261-166. 
Magurran, A. E. 1988. Ecological diversity and its measurement. Princeton Univ. Press, Princeton, (New Jersey). Pp. 1-179.

Motta-Junior, J. C., M. A. Monteiro Granzinoli, and P. F. Develey. 2008. Aves da estação ecológica de Itirapina, estado de São Paulo, Brasil. Biota Neotrop 8(3):207-227.

Oliver, I., and A. J. Beattie. 1993. A possible method for the rapid assessment of biodiversity. Conservat Biology 7: 562-568.

Orjuela, O. J., and G. Jiménez. 2004. Estudio de la abundancia relativa para mamíferos en diferentes tipos de coberturas y carretera, finca hacienda cristales, área cerritos La Virginia, Municipio de Pereira, departamento de Risaralda, Colombia. Rev. Facultad de Ciencias 9:87-96.

Pereyra, F. 2003. Ecorregiones de la Argentina. Servicio Geológico Minero Argentino, Buenos Aires, Argentina. Pp. 182.

Ramírez, M. J. 1999. Orden Araneae. Pp. 107 en: F. A. Crespo, M. S. Iglesias and A. C. Valverde (eds.). El ABC en la determinación de artrópodos. Claves para especímenes presentes en la Argentina I. Editorial CCC Educando, Buenos Aires.

Rubio, G. 2015. Diversidad de arañas (Araneae, Araneomorphae) en la Selva De Montaña: un caso de estudio en las Yungas argentinas. Graellsia 71(2):1-21.

Rubio, G. D., J. A. Corronca, and M. P. Damborsky. 2008. Do spider diversity and assemblages change in different contiguous habitats? A case study in the protected habitats of the Humid Chaco ecoregion, north-east Argentina. Environ Entomol 37:419-430.

Tóthmérész, B. 1995. Comparison of different methods for diversity ordering. J Vegetation Science 6:283-290.

Toft, S. 1989. Aspects of the ground-living spider fauna of two barley fields in Denmark: species richness and phonological synchronization. Entomologiske Meddelelser 57:157-168.

Uetz, G. W. 1991. Habitat structure and spider foraging. Pp. 325-348 en: S. S. Bell, E. D. McCoy and H. R. Mushinsky (eds.). Habitat Structure: The physical arrangement of objects in space. Chapman, and Hall, Londres, Inglaterra.

Weeks, R. D., and T. O. Holtzer. 2000. Habitat and Season in Structuring Ground-Dwelling Spider (Araneae) Communities in a Shortgrass Steppe Ecosystem. Environ Entomol 29:1164-1172.

Weyland, F., and M. E. Zaccagnini. 2008. Efecto de las terrazas sobre la diversidad de artrópodos caminadores en cultivos de soja. Ecología Austral 18:357-366.

Wise, D. H. 1993. Spiders in Ecological Webs. Cambridge, Cambridge, Inglaterra. Pp. 328.

World Spider Catalog. 2016. World Spider Catalog. Natural History Museum Bern, online at http://wsc.nmbe.ch, version 17 (visitado el 15 de enero de 2016). 